OPEN ACCESS

Edited by:

Ludovico Abenavoli,

University of Catanzaro, Italy

Reviewed by:

Gaia Chiara Mannino,

University of Catanzaro, Italy

Oksana Zayachkivska,

Danylo Halytsky Lviv National Medical University, Ukraine

*Correspondence:

Hongwen $\mathrm{Li}$

hongwenli12@163.com

Xiaoming Fan

fanxiaom1987@glmc.edu.cn

${ }^{\dagger}$ These authors have contributed equally to this work

Specialty section:

This article was submitted to

Gastrointestinal and

Hepatic Pharmacology,

a section of the journal

Frontiers in Pharmacology

Received: 21 November 2019

Accepted: 08 April 2020

Published: 29 April 2020

Citation:

Zhang G, Cai X, He L, Qin D, Li H and Fan X (2020) Skimmin Improves Insulin

Resistance via Regulating the Metabolism of Glucose: In Vitro and In Vivo Models.

Front. Pharmacol. 11:540. doi: 10.3389/fphar.2020.00540

\section{Skimmin Improves Insulin Resistance via Regulating the Metabolism of Glucose: In Vitro and In Vivo Models}

\author{
Guoqiang Zhang ${ }^{1,2 \dagger}$, Xin $\mathrm{Cai}^{1 \dagger}$, Lingmin $\mathrm{He}^{1}$, Dingmei Qin ${ }^{3}$, Hongwen $\mathrm{Li}^{2 *}$ \\ and Xiaoming Fan ${ }^{2 \times t}$ \\ ${ }^{1}$ School of Biotechnology and Food Engineering, Anyang Institute of Technology, Anyang, China, ${ }^{2}$ Department of Human \\ Anatomy, College of Basic Medical Sciences, Guilin Medical University, Guilin, China, ${ }^{3}$ College of Pharmaceutical Science, \\ Yunnan University of Chinese Medicine, Kunming, China
}

Skimmin is the major pharmacologically active component present in Hydrangea paniculata, in the traditional Chinese medicine as an anti-inflammatory agent, and its anti-inflammation and anti-diabetic effect has had been studied in previous studies. The metabolism of glucose plays an important role in the pathophysiology of diabetes. Therefore, it was identified as an important target for improving diabetic. Herein, we found that skimmin relieved the palmitic acid and high-fat and high sugar-induced insulin resistance. Furthermore, skimmin enhanced the glucose uptake via inhibiting reactive oxygen species (ROS) and reducing the level of inflammatory correlation factor. Meanwhile, skimmin reduced the glucose output by promoting PI3K/Akt signaling pathway and down-regulating the expression of glycogen synthase kinase-3 $\beta$ (GSK3 $\beta$ ) and glucose-6-phosphatase (G6Pase). In conclusion, skimmin can improve the insulin resistance by increasing glucose uptake and decreasing glucose output in vitro and in vivo.

Keywords: skimmin, glucose metabolism, palmitic acid, high fat and high sugar, insulin resistance

\section{INTRODUCTION}

Type 2 diabetes is a group of metabolic diseases which characterized by multiple etiologies caused chronic hyperglycemia. The main cause of type 2 diabetes is insulin resistance. Insulin resistance means the physiological doses of insulin cannot get their normal biological effects, and decrease the uptake and utilization of glucose (Sathya Bhama et al., 2012). The previous studies indicated that the number of people with diabetes in China have reached 92.4 million in 2010 (Yang et al., 2010). The people of diabetes will be reached 336 million in 2030 (Wild et al., 2004).

The establishment of insulin resistance model opens a window to study diabetes. Palmitic acid was usually used to induce the model of insulin resistance in HepG2 cells (Chen et al., 2019).

Abbreviations: ALT, alanine aminotransaminase; AST, aspartate transaminase; MAPK, mitogen-activated protein kinase; NF- $\mathrm{KB}$, nuclear factor kappa-B; ERKs, extracellular regulated protein kinases; JNKs, c-Jun N-terminal kinases; PI3K, Phosphoinositide-3 kinase; NOX3, NADPH oxidase 3; GSK3H, glycogen synthase kinase-3o; G6pase, glucose-6-phosphatase; MTS, 3-(4,5dimethylthiazol-2-yl)-5-(3-carboxymethoxyphenyl)-2H-tetrazdium; DCFH-DA, 2',7'-Dichlorofluorescin diacetate. 
Meanwhile, high fat and high sugar were utilized to induce the model of insulin resistance in SD rats (Fan et al., 2019a). With these models, it becomes more easier to research the effectiveness of drugs. Due to the low toxicity of natural drugs, more and more people are competing for prevention and treating type 2 diabetes.

Previous study showed that skimmin can improve membranous glomerulonephritis through suppressing inflammation and immune complex deposition (Zhang et al., 2013). Skimmin also can inhibit the streptozotocin-induced diabetic nephropathy in Wistar rats (Zhang et al., 2012). However, the molecular mechanism of skimmin for suppressing insulin resistance has not been reported before.

In our study, this is the first study to investigate the skimmin can increase glucose uptake, promote glycogen synthesis and improve insulin resistance in vitro and in vivo.

\section{MATERIALS AND METHODS}

\section{Cell and Animal Model Building}

The HepG2 cells were purchased from ATCC, Virginia, USA. It was cultured in Dulbecco's modified Eagle's medium (DMEM) with $10 \%$ fetal bovine serum (FBS) at a $37^{\circ} \mathrm{C}$ incubator containing $5 \% \mathrm{CO}_{2}$. HepG2 cells were exposed in different dose of palmitic acid $(0.1,0.2,0.3,0.4 \mathrm{mmol} / \mathrm{L})$ for different time $(24,36,48,60 \mathrm{~h})$. Then they were administrated with different concentration of skimmin $(10,20,40 \mu \mathrm{M})$ for $24 \mathrm{~h}$. The glucose content of medium was tested by glucose oxidase kit. The optimal dosage and time of palmitate were determined according to the glucose concentration of medium.

Adult Sprague Dawley rats $(6-8 \mathrm{~W})(\mathrm{n}=50)$ were purchased in Laboratory Animal Center of Zhengzhou University. The experiment of animals was approved by the Institutional Animal Care and Use Committee of Anyang Institute of Technology (IACUC approval no. 2018-001). The animals were anesthetized with $10 \%$ chloral hydrate and sacrificed by cervical dislocation. All efforts were made to minimize suffering. At first, The animals were randomly divided into two group: normal diet group $(n=10)$ and HFHS diet group $(n=40)$. The normal group was basic diet. The HFHS diet group was feed with $60 \%$ basic diet, $20 \%$ lard, $15 \%$ refined sugar, $1.5 \%$ cholesterol, $0.1 \%$ sodium cholate, and $3.4 \%$ peanuts. After 12 weeks of dietary manipulation, the 40 rats were again randomly divided into 4 groups: HFHS group $(\mathrm{n}=10)$, Low dose $(10 \mathrm{mg} / \mathrm{kg} / \mathrm{d})$ skimmin group $(\mathrm{n}=10)$, Middle dose $(25 \mathrm{mg} / \mathrm{kg} / \mathrm{d})$ skimmin group $(\mathrm{n}=10)$, High dose $(50 \mathrm{mg} / \mathrm{kg} / \mathrm{d})$ skimmin group $(\mathrm{n}=10)$. Each group was administrated for 4 weeks.

\section{Glucose, Insulin, and Hepatic Function Assay}

The glucose concentration of medium and blood were detected by the kit of glucose oxidase-peroxidase (Baoping Bioengineering Institute, Zhengzhou). Glycogen levels in medium and liver tissue were measured via the kit of Glycogen Assay (Baoping Bioengineering Institute, Zhengzhou) according to the instructions of manufacturer. The levels of TNF- $\alpha$, IL-6, insulin, and IL-1 $\beta$ were detected by ELISA kits (Baoping Bioengineering Institute, Zhengzhou) following the instructions of manufacturer. Alanine transaminase (ALT) and aspartate transaminase (AST) were determined by the automated biochemistry analyzer.

\section{MTS Assay}

Skimmin was obtained from the Zhengzhou Baoping Biological Technology Co. LTD (Zhengzhou, China). The purity of skimmin is $98.5 \%$. After the cells were treated with different concentration of skimmin, the cytotoxicity of skimmin was detected by MTS (3-(4,5-dimethylthiazol-2-yl)-5-(3carboxymethoxyphenyl)-2H-tetrazdium) assay kit according to the instruction.

\section{DCFH-DA Staining Combined With Flow Cytometry Assay}

The cells were seed $\left(1^{\star} 10^{5}\right)$ in 6 -well plates and cultured overnight, and then were fed with serum-free medium containing DCFH-DA (1:8000). Then, the cells were continuously cultured for $30 \mathrm{~min}$ in the incubator and washed with PBS for two times, the cells were collected and filtered with 200 using mesh screen. Then the intracellular ROS levels were determined by flow cytometry according to our previous research methods (Fan et al., 2019b).

\section{Western Blot}

The protein of cells was extracted by RIPA lysate, and its concentration were determined by the BCA method. The samples were loaded to $10 \%$ SDS-PAGE gel and transferred onto polyvinylidene fluoride (PVDF) membrane. After the membrane was blocked by $5 \%$ skim milk, it was incubated with a specific primary antibody against p38 MAPK, JNKs, NF- $\mathrm{B}$, PI3K, Akt, G6Pase, phosphorylated p38 MAPK (Thr180/Thr182), phosphorylated JNKs (Thr182/Thr185), phosphorylated NF-KB (Ser 536), phosphorylated PI3K (Tyr458/Tyr199), phosphorylated Akt (Ser 473), and $\beta$-actin at $4^{\circ} \mathrm{C}$ overnights. All above antibody dilution concentration is 1:1000. Then, the membrane was incubated with the horseradish peroxidase (HRP)-conjugated secondary antibody (1:3000). The strip was visualized by enhanced chemiluminescence (ECL) kit and quantified using Image J 12.0 software.

\section{Immunohistochemistry Staining}

The tissue sections $(5 \mu \mathrm{m})$ was performed antigen retrieval by microwave after deparaffinization and rehydration for $10 \mathrm{~min}$ in sodium citrate buffer. Sections were cooled to room temperature, treated with $3 \% \mathrm{H}_{2} \mathrm{O}_{2}$ for $10 \mathrm{~min}$ and blocked with $5 \%$ goat serum $40 \mathrm{~min}$ at room temperature. The sections were incubated at 4 locked with $5 \%$ goat serum $40 \mathrm{~min}$ at room temperature. The sections weodium citrate buffer. Sections were cooled to room temperature, treated with $3 \%$ Hnt target for improving diabetic. Herei-rabbit, diluted 1:200) for $30 \mathrm{~min}$. The sections were counterstained with hematoxylin after diaminobenzidine 
staining according to our previous research methods (Fan et al., 2019a).

\section{Statistical Analysis}

Data were expressed as the mean ch methods $40 \mathrm{~min}$ at room temperature. The sections weodium citrate buffer. Sections were cooled to room temperature, treated with 3\% Hnt target for improving diabetic. Heresis of variance. $P<0.05$ was considered to indicate a statistically significant difference.

\section{RESULTS}

\section{The Model of Insulin Resistance Is Built In Vitro and In Vivo}

The cells model of insulin resistance was established using palmitic acid to induce HepG2 cells. The cells were treated in different dose of palmitic acid $(0.1,0.2,0.3,0.4,0.5 \mathrm{mmol} / \mathrm{l})$ for different time $(24,36,48,72 \mathrm{~h})$. Next, the glucose content in medium was detected by glucose oxidase kit. The results indicated that the glucose consumption was highest in the 0.2 $\mathrm{mmol} / \mathrm{L}$ of palmitic acid. In addition, when $0.2 \mathrm{mmol} / \mathrm{L}$ palmitic acid caused the highest blood glucose consumption at $36 \mathrm{~h}$ (Figures 1A, B). Therefore, the insulin resistance cell model was established under the following conditions: $0.2 \mathrm{mmol} / \mathrm{L}$ palmitic acid for $36 \mathrm{~h}$.

Besides, the animal model of insulin resistance was established by high fat and high sugar. The results showed that the levels of glucose, insulin, and insulin resistance index (HOMA-IR) were increased in the model group compared with the control group (Figures 1C-E), which means the animal model of insulin resistance was built successfully.

\section{Skimmin Reduce Blood Glucose and Improve Insulin Resistance In Vitro and In Vivo}

The chemical structure of skimmin is shown in Figure 2A. MTS assay results showed that skimmin had no cytotoxicity to HepG2 cells (Figure 2B).

Then, we investigated whether skimmin had an effect on the glucose consumption of palmitic acid-induced HepG2 cells. The results showed that skimmin promoted the absorption of glucose in a dose dependent manner in palmitic acid-induced HepG2 cells. Metformin was used as the positive control group (Figure 2C).

Furthermore, the in vivo studies showed that skimmin decreased the level of serum glucose, insulin, and improved HOMA-IR (Figures 2D-F). Furthermore, we found that skimmin can decrease liver weight, body weight, and ratio of them induced by high fat and high sugar (Figures 2G-I). Besides, HE staining showed that skimmin inhibited the pathological changes of liver induced by high fat and high sugar (Figure 2J). Meanwhile, skimmin suppressed the secretion of lipid factors (Figure $\mathbf{2 K}$ ), and improved the function of liver in a dose dependent manner (Figure 2L).

\section{Skimmin Increase the Uptake of Glucose by Reducing the Activation of Inflammatory Signaling and Inhibiting Oxidative Stress In Vitro and In Vivo}

Nowadays, we have known that skimmin can promote the uptake of glucose to improve insulin resistance in vitro and in vivo. However, the molecular mechanism by which skimmin reduce blood glucose is still unclear. Oxidative stress is the pathological basis of insulin resistance (Cremonini and Oteiza, 2018; Dos Santos et al., 2018). The activity of the NADPH oxidase (NOX) is critical to the production of ROS in the organism. Previous studies showed that the ROS production increased by up-regulating the expression of NADPH oxidase 3 (NOX3), activating p38MAPK and JNK signaling pathway, and inducing insulin resistance in palmitate-induced HepG2 cells (Gao et al., 2010; Malik et al., 2019). Our studies found that skimmin can inhibit palmitic acid-induced the production of ROS, and $40 \mu \mathrm{mol} / \mathrm{L}$ skimmin was more obvious using flow cytometry assay $(P<0.05)$, which was better than metformin, a drug used to treat diabetes (Figure 3A). In addition, skimmin also inhibited the increased of NOX3 protein compared with the insulin resistance group induced by palmitic acid. The effect of 40 $\mu \mathrm{M}$ skimmin was better than metformin (Figure 3B) $(P<0.05)$. What is more, we found that skimmin reduced the phosphorylation expression of p38MAPK and JNKs compared with insulin resistance group in a dose dependent manner in vitro and in vivo (Figures $3 \mathrm{C}, \mathrm{D}$ ). AP-1 transcription factors, including c-Fos, c-Jun, and ATF, which also are the down-stream of p38 and JNKs, have a well-known role in promoting IL-6 and TNF- $\alpha$ transcription (Oh et al., 2013; MacNeil et al., 2014). In order to further detect the anti-inflammatory mechanism of skimmin, we tested the expression of NF- $\mathrm{BB}$ and the secretion of inflammatory factors. Our studies indicated that skimmin inhibited the phosphorylation level of NF- $\kappa \mathrm{B}$ and the secretion of IL- 6, IL- $1 \beta$, and TNF- $\alpha$ in a dose dependent manner in vitro and in vivo (Figure 3E, F). The above results showed that skimmin can promote the uptake of glucose and reduce inflammation caused by insulin resistance through reducing the production of ROS and suppressing the expression of NOX3, p-p38MAPK, p-JNKs, and NF- $\kappa B$.

\section{Skimmin Improve the Synthesis of Liver Glycogen by Increasing the Phosphorylation of Akt and Inhibiting the Expression of GSK3 $\beta$ In Vitro and In Vivo}

GSK3 $\beta$ is a critical enzyme which can reduce the synthesis of liver glycogen, increase the concentration of blood sugar in the body (Zhang et al., 2018). Some studies indicated that Grifola Frondosa stimulates glycogen synthesis by regulating PI3K/Akt/GSK3 signaling pathway, improving insulin resistance (Ma et al., 2014). To further explore the relationship between skimmin and glycogen synthesis. The PI3K/Akt/GSK3 signaling pathway was determined by Western blot after skimmin treatment in vitro and in vivo. The results demonstrated that skimmin can obviously upregulate the level of p-PI3K, p-Akt and downregulate the level of GSK3 $\beta$ 


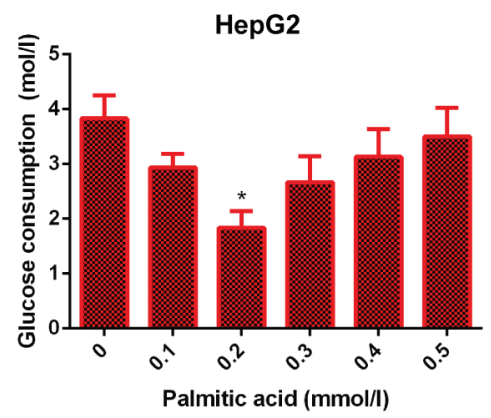

C

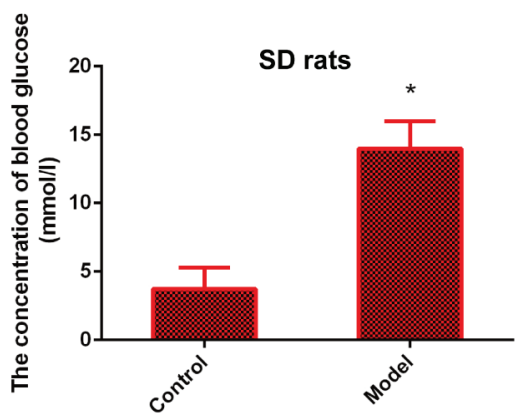

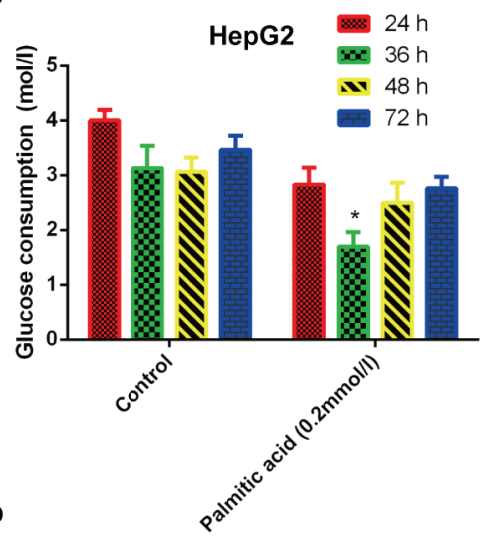

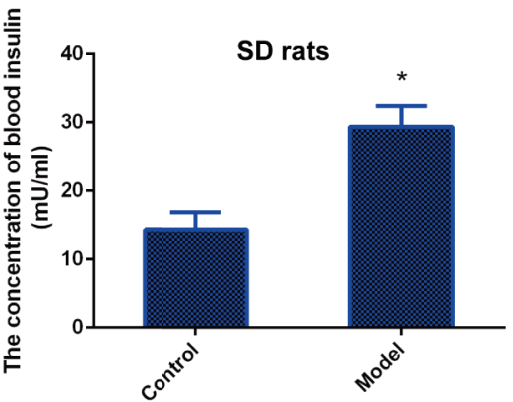

$\mathbf{E}$

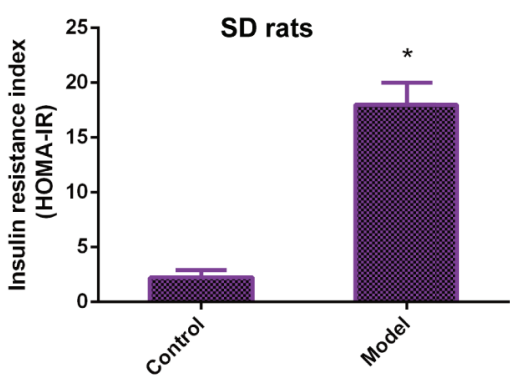

FIGURE 1 | The model of insulin-resistance is built in vitro and in vivo. (A) Effects of different concentrations of palmitic acid on glucose consumption. (B) Effects of different time of palmitic acid on glucose consumption. (C-E). High fat and high sugar induced the insulin resistance model in vivo. Insulin resistance index (HOMA-IR) were calculated by formula INS*GLU/22.5. *Significant compared with control group alone, $P<0.05$. LD, low dose; MD, middle dose; HD, high dose.

compared with insulin resistance model group in a dose dependent manner in vitro and in vivo (Figures $4 \mathbf{A}, \mathbf{B}$ ).

Previous studies showed that GSK3 $\beta$ inhibitor can suppress the synthesis of glycogen (Maqbool and Hoda, 2017). Furthermore, the volume of glycogen was detected by glycogen assay kit. We found that glycogen was decreased in the model group, while skimmin can increase the volume of glycogen in a dose dependent manner in vitro (Figure 4C). G6pase increases the blood glucose concentration in the body by promoting the decomposition of glycogen (Chou et al., 2015). Immunohistochemical and Western blot results demonstrated that skimmin inhibit the expression of G6pase in a dose dependent manner in vivo (Figures 4D, E).
The above results showed that skimmin can decrease glucose output by up-regulating the phosphorylation level of PI3K and Akt, suppressing the expression of GSK3, promoting the synthesis of glycogen. Meanwhile, skimmin also can decrease the expression of G6pase, inhibit the breakdown of glycogen.

\section{DISCUSSION}

The decomposition of glycogen can increase blood glucose, which is then decomposed and used by cells. The excess blood glucose is converted into glycogen, maintaining the balance of 
<smiles>O=c1ccc2ccc(O[C@@H]3O[C@H](CO)[C@@H](O)[C@H](O)C3O)cc2o1</smiles>

D

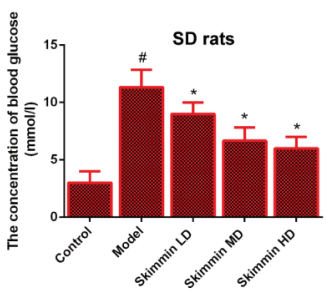

G

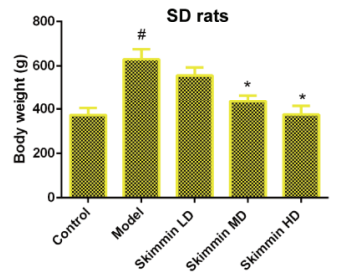

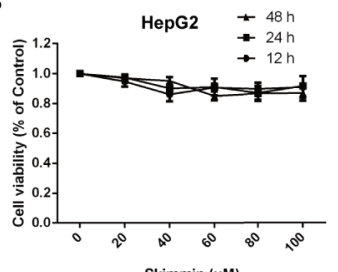

E

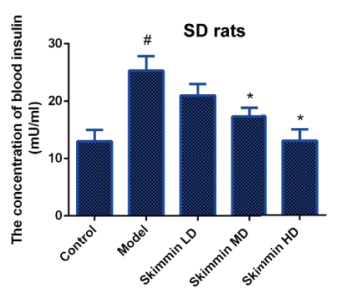

H

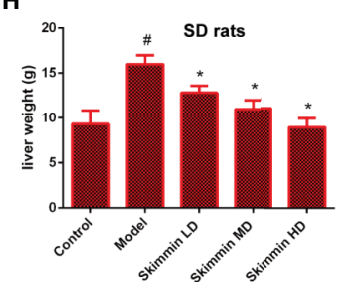

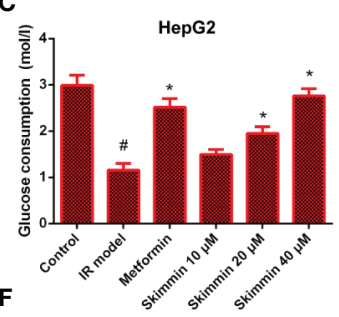

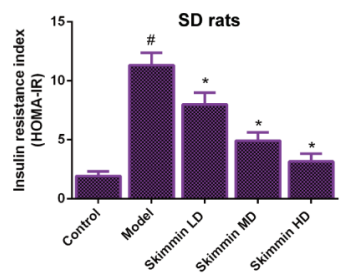

I

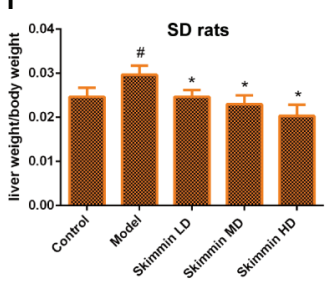

J
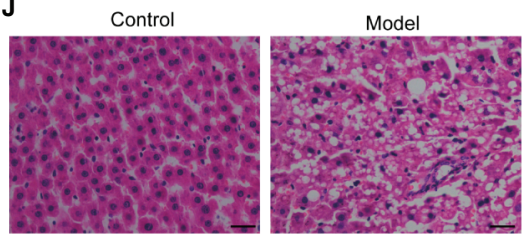

Skimmin LD

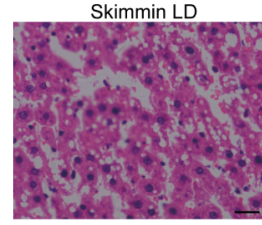

Skimmin MD

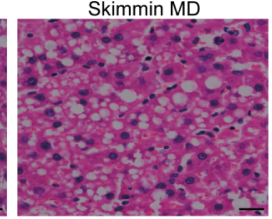

Skimmin HD
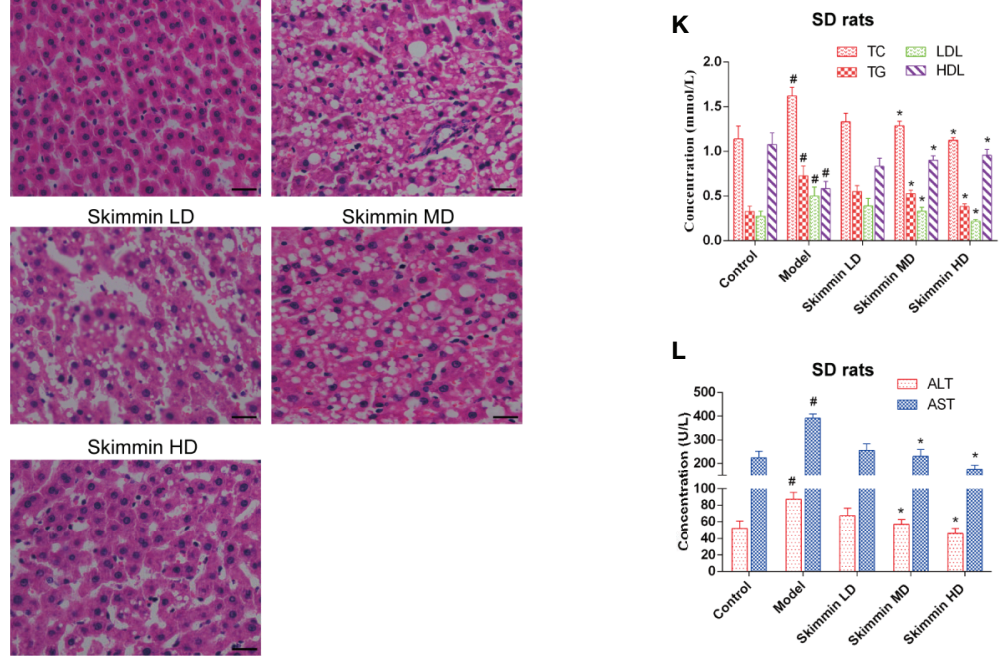

L

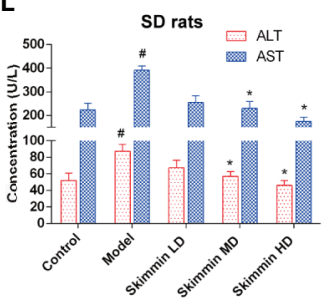

FIGURE 2 | Skimmin reduce blood glucose and improve insulin resistance in vitro and in vivo. (A) The chemical structure of skimmin. (B) Cytotoxic effect of skimmin on HepG2 cells. (C) Skimmin promotes the uptake of glucose. (D-F). Skimmin inhibits insulin resistance in a dose dependent manner. (G-I) Skimmin decrease the body weight and liver weight of rat induced by high fat and high sugar. (J) Skimmin attenuates the pathologic change of liver tissues. Bar $=20$ um. (K) Ginsenoside Rg1 reduces the concentration of serum lipid under the condition of insulin resistance. (L) Ginsenoside Rg1 improve the function of liver under the condition of insulin resistance. "Significant compared with control group alone $(P<0.05)$. *Significant compared with insulin resistance group alone $(P<0.05)$. TC, triglyceride; TG, total cholesterol; LDL, low-density lipoprotein; HDL, high-density lipoprotein; ALT, alanine transaminase; AST, aspartate transaminase.

blood glucose in the body under the regulation of insulin and glucagon. Under the condition of insulin, insulin of normal concentration cannot play its due role, thus causes the increase of blood sugar (Yang et al., 2011; Tangvarasittichai, 2015). We explored the molecular mechanism of insulin resistance from two aspects: the utilization of blood glucose and the decomposition of glycogen.

Oxidative stress leads to the production of a large number of ROS, which further leads to the apoptosis of islet cells, and then cause insulin resistance (Hu et al., 2016; Kuzmenko et al., 2016; 


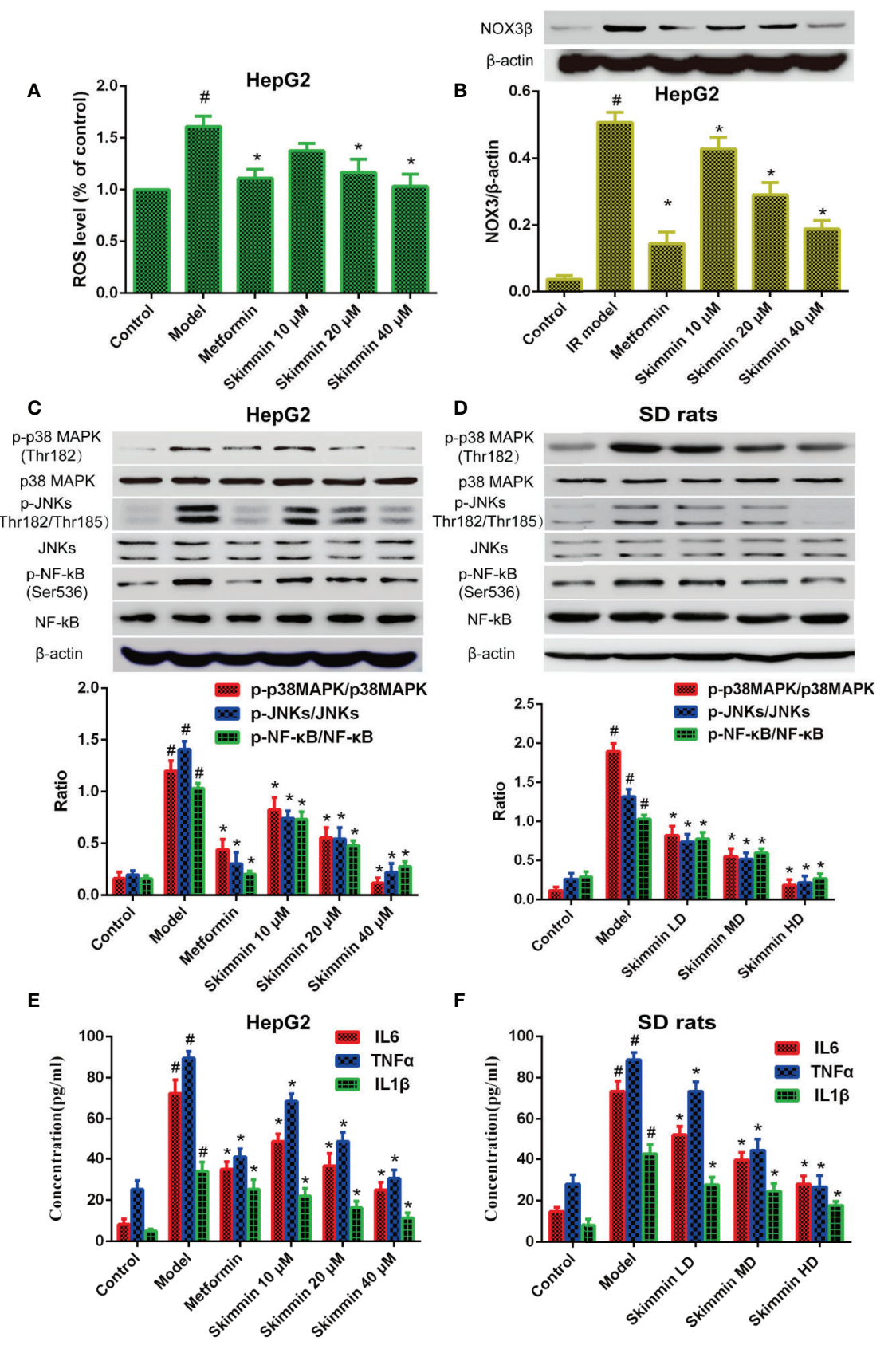

FIGURE 3 | Skimmin increase the uptake of glucose by reducing the activation of inflammatory signaling and inhibiting oxidative stress in vitro and in vivo. (A) Insulin promote the production of ROS in insulin resistance HepG2 cells. (B) Skimmin inhibit the expression of NOX3 in a dose dependent manner. (C, D) Skimmin inhibit the phosphorylation expression of p38 MAPK, JNKs, and NF- $\mathrm{KB}$ in a dose-dependent manner in vitro and in vivo. (E, F) Skimmin inhibits the secretion of inflammatory cytokines under the condition of insulin resistance in vitro and in vivo. The values shown are mean SEM of data from three independent experiments. \#Significant compared with control group alone, $P<0.05$. *Significant compared with model group alone, $P<0.05$. ROS, reactive oxygen species; NOX3, NADPH oxidase.

Alcalá, et al., 2017). Previous studies showed that TNF- $\alpha$ induced the expression of NOX3, promotes ROS production, activates the JNKs signaling pathway, and produces insulin resistance in the HepG2 cells (Walton, 2017). What is more, high sugar and ROS also can activate p38MAPK and induce insulin resistance in vascular smooth muscle (Liu et al., 2018). Therefore, it is necessary to find the agent to decrease ROS, suppress the secretion of inflammatory factors. enhance islet cell secreting function, increase the utilization of blood glucose and reduce blood glucose. 
C
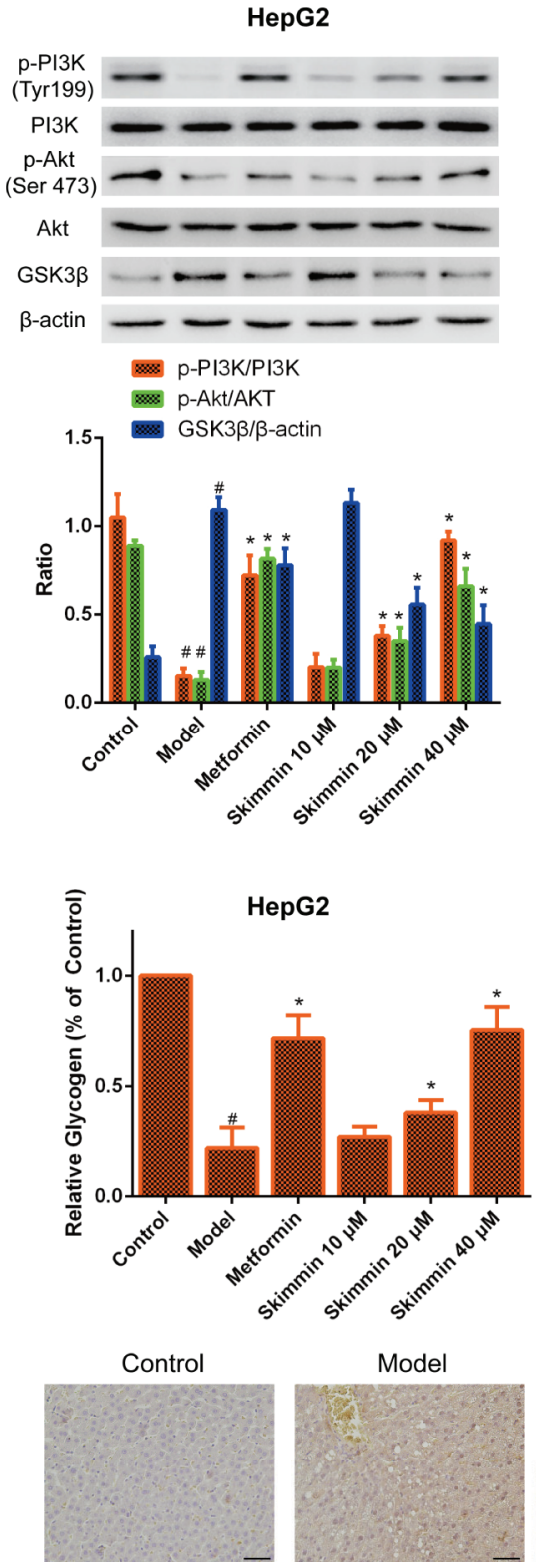

Skimmin MD
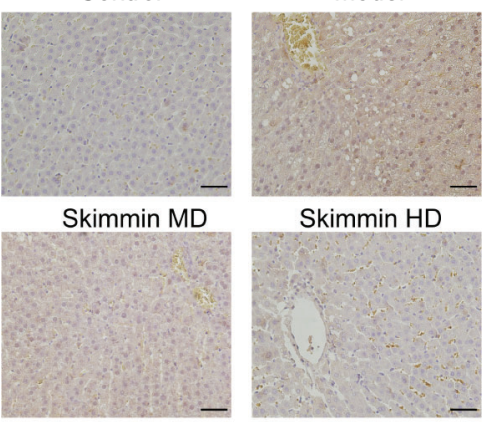

Skimmin HD

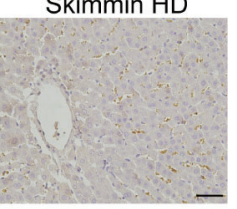

B

SD rats

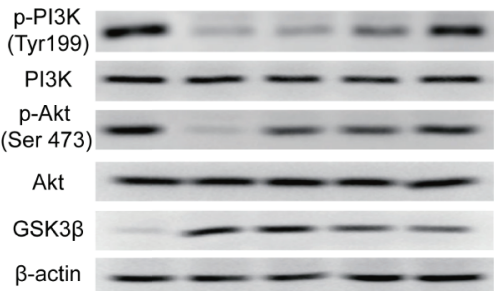

D
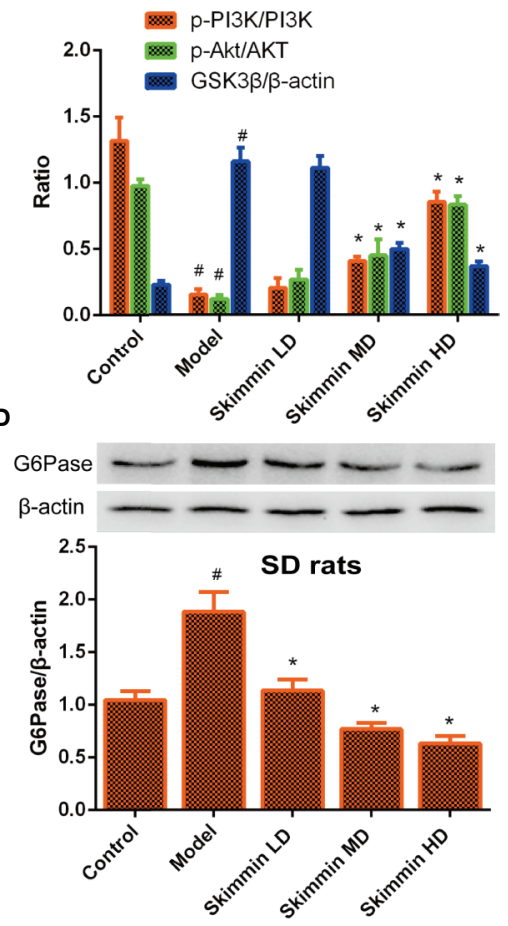

Skimmin LD
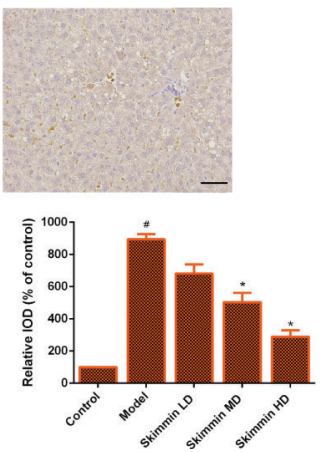

FIGURE 4 | Skimmin increase the phosphorylation of Akt, inhibit the expression of GSK3ß, and increase the synthesis of liver glycogen in vitro and in vivo. (A, B) Skimmin upregulate the protein expression of phosphor-PI3K, phosphor-AKt and downregulate the protein expression of GSK3 $\beta$ in a dose dependent manner in vitro and in vivo. (C). Skimmin increases the synthesis of glycogen in a dose dependent manner in vitro. (D, E) Skimmin inhibits the expression of G6Pase in a dose dependent manner in vivo. Bar $=20 \mu \mathrm{m}$. "Significant compared with control group alone $(P<0.05)$. *Significant compared with insulin resistance group alone

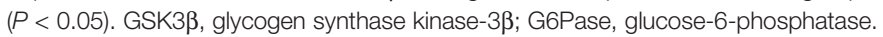




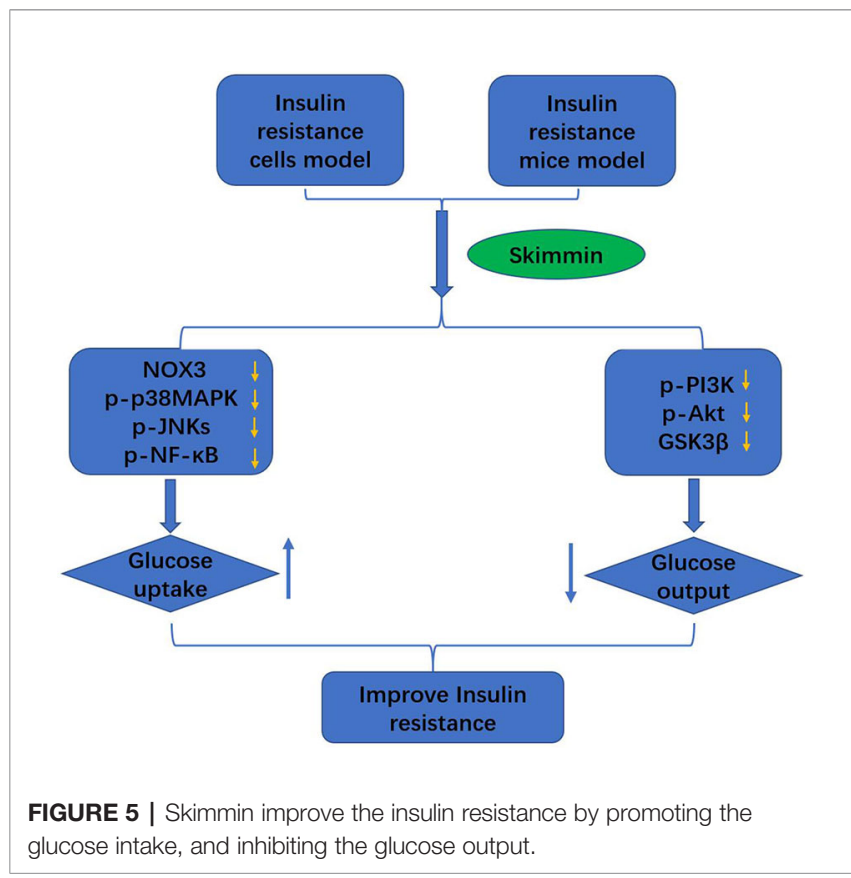

In addition, the glycogen synthesis also plays a critical role in the glucose output of the liver. Previous studies indicated that the enhancement of glycogenesis and decrease in glycogen synthesis are also responsible for insulin resistance (Ren et al., 2018). The PI3K/ Akt/GSK3 signaling pathway is involved in the metabolism of glycogen. The activation of PI3K is required for insulin-stimulated glucose uptake (Yang et al., 2015). Phosphorylated Akt can inhibit the activity of GSK3, and GSK3 can suppress synthesis of glycogen by inhibiting glycogen synthase (Yang et al., 2017). Furthermore, G6pase is a critical enzyme in gluconeogenesis and glycogenolysis and plays an important role in the homeostasis of glucose (Chou et al., 2015). Inhibiting the expression of G6pase can also reduce blood glucose. So, it is also important to find drugs to inhibit the production of glucose through upregulating the expression of $\mathrm{p}-\mathrm{AKt}$, downregulating GSK3 expression, and inhibiting G6pase expression.

Skimmin is the main active substance in Hydrangea paniculata, and has the characteristics of anti-inflammation, anti-plasmodial, and anti-cancer (Moon et al., 2011). We build the palmitic acidinduced HepG2 insulin resistance cell model and high fat and high sugar-induced insulin resistance SD rat model. Then skimmin was used to treat the HepG2 cells and SD rats, metformin as a control

\section{REFERENCES}

Alcalá, M., Calderon-Dominguez, M., Bustos, E., Casals, N., Serra, D., et al. (2017). Increased inflammation, oxidative stress and mitochondrial respiration in brown adipose tissue from obese mice. Sci. Rep. 7 (1), 16082. doi: 10.1038/s41598-017-16463-6

Chen, L., Sun, X., Xiao, H., Xu, F., Yang, Y., Lin, Z., et al. (2019). PAQR3 regulates phosphorylation of FoxO1 in insulin-resistant HepG2 cells via NF- $\kappa B$ signaling pathway. Exp. Cell Res. (19), 30222-30228. doi: 10.1016/j.yexcr.2019.04.031

Chou, J. Y., Jun, H. S., and Mansfield, B. C. (2015). Type I glycogen storage diseases: disorders of the glucose-6-phosphatase/glucose-6-phosphate transporter complexes. J. Inherit. Metab. Dis. 38 (3), 511-559. doi: 10.1007/ s10545-014-9772-x agent. The results showed that skimmin increased glucose intake and suppressed the inflammatory response through decreasing the production of ROS, suppressing the protein expression of NOX3, pp38MAPK, and p-JNKs. Meanwhile, skimmin decreased glucose output by increasing the phosphorylated PI3K and Akt, suppressing the expression of GSK3 $\beta$, reducing the glycogen synthase, increase liver glycogen synthesis, and inhibiting G6pase expression, reducing decomposition of glycogen (Figure 5). The above will provide better drug options for the treatment of type 2 diabetes. To validate the utility of skimmin for type 2 diabetes patient improvement, more detailed investigation regarding pharmacokinetics of skimmin following its overdose should be addressed. In addition, the effectiveness of skimmin on the relevance markers of insulin resistance need to be further explored.

Taken together, skimmin improve eventually the insulin resistance by promoting the glucose intake, and inhibiting the glucose output.

\section{DATA AVAILABILITY STATEMENT}

All datasets generated for this study are included in the article/ supplementary material.

\section{ETHICS STATEMENT}

The animal study was reviewed and approved by Anyang Institute of Technology.

\section{AUTHOR CONTRIBUTIONS}

$\mathrm{XF}$ and HL designed the research, GZ and XC performed the research and wrote the paper. $\mathrm{LH}$ and DQ analyzed the data.

\section{ACKNOWLEDGMENTS}

This work was supported by grants from Henan provincial science and technology research fund [183400410067]. 
Fan, X., Tao, J., Zhou, Y., Hou, Y., Wang, Y., Gu, D., et al. (2019b). Investigations on the effects of ginsenoside-Rg1 on glucose uptake and metabolism in insulin resistant HepG2 cells. Eur. J. Pharmacol. 843, 277-284. doi: 10.1016/j.ejphar.2018.11.024

Gao, D., Nong, S., Huang, X., Lu, Y., Zhao, H., Lin, Y., et al. (2010). The effects of palmitate on hepatic insulin resistance are mediated by NADPH Oxidase 3derived reactive oxygen species through JNK and p38MAPK pathways. J. Biol. Chem. 285 (39), 29965-29973. doi: 10.1074/jbc.M110.128694

Hu, Y., Hou, Z., Liu, D., and Yang, X. (2016). Tartary buckwheat flavonoids protect hepatic cells against high glucose-induced oxidative stress and insulin resistance via MAPK signaling pathways. Food Funct. 7 (3), 1523-1536. doi: 10.1039/C5FO01467K

Kuzmenko, D. I., Udintsev, S. N., Klimentyeva, T. K., and Serebrov, V. Y. (2016). Oxidative stress in adipose tissue as a primary link in pathogenesis of insulin resistance. BioMed. Khim. 62 (1), 14-21. doi: 10.18097/PBMC20166201014

Liu, Y., Li, X., Jiang, S., and Ge, Q. (2018). Tetramethylpyrazine protects against high glucose-induced vascular smooth muscle cell injury through inhibiting the phosphorylation of JNK, p38MAPK, and ERK. J. Int. Med. Res. 46 (8), 3318-3326. doi: 10.1177/0300060518781705

Ma, X., Zhou, F., Chen, Y., Zhang, Y., Hou, L., Cao, X., et al. (2014). A polysaccharide from Grifola frondosa relieves insulin resistance of HepG2 cell by Akt-GSK-3 pathway. Glycoconj. J. 31 (5), 355-363. doi: 10.1007/s10719-014-9526-X

MacNeil, A. J., Junkins, R. D., Wu, Z., and Lin, T. J. (2014). Stem cell factor induces AP-1-dependent mast cell IL-6 production via MAPK kinase 3 activity. J. Leukoc Biol. 95, 903-915. doi: 10.1189/jlb.0713401

Malik, S. A., Acharya, J. D., Mehendale, N. K., Kamat, S. S., and Ghaskadbi, S. S. (2019). Pterostilbene reverses palmitic acid mediated insulin resistance in HepG2 cells by reducing oxidative stress and triglyceride accumulation. Free Radic. Res. 53 (7), 815-827. doi: 10.1080/10715762.2019.1635252

Maqbool, M., and Hoda, N. (2017). GSK3 Inhibitors in the Therapeutic Development of Diabetes, Cancer and Neurodegeneration: Past, Present and Future. Curr. Pharm. Des. 23 (29), 4332-4350. doi: 10.2174/1381612823666170714141450

Moon, H. I., Lee, J. H., Lee, Y. C., and Kim, K. S. (2011). Antiplasmodial and cytotoxic activity of Coumarin derivatives from dried roots of Angelica gigas Nakai in vitro. Immunopharmacol. Immunotoxicol. 33 (4), 663-666. doi: 10.3109/08923973.2011.559248

Oh, J., Kim, J. H., Park, J. G., Yi, Y. S., Park, K. W., Rho, H. S., et al. (2013). Radical scavenging activity-based and AP-1-targeted anti-inflammatory effects of lutein in macrophage-like and skin keratinocytic cells. Mediators Inflamm. 2013, 787042. doi: 10.1155/2013/787042

Ren, Z., Xie, Z., Cao, D., Gong, M., Yang, L., Zhou, Z., et al. (2018). C-Phycocyanin inhibits hepatic gluconeogenesis and increases glycogen synthesis via activating Akt and AMPK in insulin resistance hepatocytes. Food Funct. 9 (5), 2829-2839. doi: 10.1039/C8FO00257F

Sathya Bhama, C. V., Balaji, S., and Seethalakshmi, A. (2012). Analysis of the Degree of Insulin Resistance in Post Menopausal Women by Using Skin Temperature Measurement and Fasting Insulin and Fasting Glucose Levels: A
Case Control Study. J. Clin. Diagn. Res. 6 (10), 1644-1647. doi: 10.7860/JCDR/ 2012/4377.2646

Tangvarasittichai, S. (2015). Oxidative stress, insulin resistance, dyslipidemia and type 2 diabetes mellitus. World J. Diabetes 6 (3), 456-480. doi: 10.4239/ wjd.v6.i3.456

Walton, E. L. (2017). Oxidative stress and diabetes: Glucose response in the cROSsfire. BioMed. J. 40 (5), 241-244. doi: 10.1016/j.bj.2017.10.001

Wild, S., Roglic, G., Green, A., Sicree, R., and King, H. (2004). Global prevalence of diabetes: estimates for the year 2000 and projections for 2030. Diabetes Care 27 (5), 1047-1053. doi: 10.2337/diacare.27.5.1047

Yang, S. H., Dou, K. F., and Song, W. J. (2010). Prevalence of diabetes among men and women in China. N. Engl. J. Med. 362 (25), 2425-2426. doi: 10.1056/ NEJMoa0908292

Yang, Z. H., Miyahara, H., and Hatanaka, A. (2011). Chronic administration of palmitoleic acid reduces insulin resistance and hepatic lipid accumulation in KK-Ay Mice with genetic type 2 diabetes. Lipids Health Dis. 10, 120. doi: 10.1186/1476-511X-10-120

Yang, M., Ren, Y., Lin, Z., Tang, C., Jia, Y., Lai, Y., et al. (2015). Krüppel-like factor 14 increases insulin sensitivity through activation of PI3K/Akt signal pathway. Cell Signal. 27 (11), 2201-2208. doi: 10.1016/j.cellsig.2015.07.019

Yang, S., Chen, Z., Cao, M., Li, R., Wang, Z., Zhang, M., et al. (2017). Pioglitazone amelior 14 increases insuliSK3 $\beta$ activation. Mol. Med. Rep. 15 (5), 2588-2594. doi: 10.3892/mmr.2017.6342

Zhang, S., Yang, J., Li, H., Li, Y., Liu, Y., Zhang, D., et al. (2012). Skimmin, a Coumarin, suppresses the streptozotocin-induced diabetic nephropathy in Wistar rats. Eur. J. Pharmacol. 692 (1-3), 78-83. doi: 10.1016/ j.ejphar.2012.05.017

Zhang, S., Xin, H., Li, Y., Zhang, D., Shi, J., Yang, J., et al. (2013). Coumarin from Hydrangea paniculata, Slows down the Progression of Membranous Glomerulonephritis by Anti-Inflammatory Effects and Inhibiting Immune Complex Deposition. Evid Based Complement Alternat. Med. 819296. doi: $10.1155 / 2013 / 819296$

Zhang, Y., Huang, N. Q., Yan, F., Jin, H., Zhou, S. Y., Shi, J. S., et al. (2018). Diabetes mellitus and Alzheimer's disease: GSK-3 $\beta$ as a potential link. Behav. Brain Res. 339, 57-65. doi: 10.1016/j.bbr.2017.11.015

Conflict of Interest: The authors declare that the research was conducted in the absence of any commercial or financial relationships that could be construed as a potential conflict of interest.

Copyright (c) 2020 Zhang, Cai, He, Qin, Li and Fan. This is an open-access article distributed under the terms of the Creative Commons Attribution License (CC BY). The use, distribution or reproduction in other forums is permitted, provided the original author(s) and the copyright owner(s) are credited and that the original publication in this journal is cited, in accordance with accepted academic practice. No use, distribution or reproduction is permitted which does not comply with these terms. 